\title{
The changing epidemiology of VanB Enterococcus faecium in Poland
}

\author{
Ewa Sadowy $^{1}$ (D) Iwona Gawryszewska ${ }^{1} \cdot$ Alicja Kuch $^{2} \cdot$ Dorota Żabicka $^{2} \cdot$ Waleria Hryniewicz $^{2}$
}

Received: 23 November 2017 / Accepted: 1 February 2018 / Published online: 13 February 2018

(C) The Author(s) 2018. This article is an open access publication

\begin{abstract}
Increasing prevalence of VanB Enterococcus faecium in Polish hospitals reported to National Reference Centre for Susceptibility Testing (NRCST) prompted us to investigate the basis of this phenomenon. Two-hundred seventy-eight $E$. faecium isolates of VanB phenotype from the period 1999 to 2010 obtained by NRCST were investigated by multilocus sequence typing (MLST) and multilocus VNTR analysis (MLVA). Localization, transferability, and partial structure of the vanB-carrying Tn1549 transposon were studied by hybridization, PCR mapping, sequencing, and conjugation. VanB isolates almost exclusively represented hospital-associated E. faecium, with a significant shift from representatives of 17/18 lineage to 78 lineage after 2005. The vanB determinant, initially located mostly on transferable plasmids of the pRUM-, pLG1-, and pRE25-replicon types, later on was found almost exclusively on the host chromosome. Fifteen different plasmid and chromosomal insertion sites were identified, typically associated with single transposon coupling sequences, mostly not observed before. Our study demonstrates the significant change in the epidemiology of VanB-E. faecium in Poland, associated with the introduction and spread of the lineage 78 of the hospital-adapted E. faecium. These data point to the importance of the lineage 78 for the spread of vancomycin-resistance, determined by the vanB gene cluster, resulting in an increasing VRE prevalence in hospitals. This study also supports the scenario, in which representatives of the hospital-associated $E$. faecium independently acquire the van $B$ determinant de novo and spread within and among hospitals, concomitantly undergoing differentiation.
\end{abstract}

Keywords Epidemic lineage $\cdot$ Population shift $\cdot$ Transposon $\cdot$ Plasmid $\cdot$ Diversity

\section{Introduction}

The importance of enterococci as etiologic agents of hospitalacquired infections (HAIs) is currently increasing [1], and common glycopeptide resistance among these bacteria is especially alarming [2]. Among two most ubiquitous van gene clusters, responsible for this phenotype, vanA confers resistance to both vancomycin and teicoplanin, and van $B$ typically determines resistance only to vancomycin [3]. The vanB

Electronic supplementary material The online version of this article (https://doi.org/10.1007/s10096-018-3209-7) contains supplementary material, which is available to authorized users.

Ewa Sadowy

ewasadowy@cls.edu.pl

1 Department of Molecular Microbiology, National Medicines Institute, ul. Chełmska 30/34, 00-725 Warsaw, Poland

2 Department of Epidemiology and Clinical Microbiology, National Medicines Institute, Chełmska 30/34, 00-725 Warsaw, Poland cluster is predominantly associated with the Tn1549-type transposons [4], which may reside either on plasmids or on the bacterial chromosome [4-9]. During the initial steps of conjugative transfer of transposon, the staggered cleavage by the Int recombinase results in the formation of a circular intermediate, joined by a 5- to 6-bp sequence originating from the donor genome, termed a coupling sequence, which, after transposition, is found adjacent to the transposon termini in the recipient [7].

Among the two clinically most important enterococcal species, i.e., Enterococcus faecalis and Enterococcus faecium, the latter is particularly prone to the acquisition of antimicrobial resistance determinants, including vanA and vanB clusters (vancomycin-resistant $E$. faecium, VREfm), resulting in increasing proportion of VREfm among hospital E. faecium [10]. Concomitantly, an increase in the incidence of HAIs caused by E. faecium is observed [10,11], likely due to the selection and worldwide dissemination of successful hospitaladapted clonal complex 17 (CC17) [12] that combines resistance to several antimicrobials with the enrichment in pathogenicity factors and increased epidemic potential. The 
Bayesian Analysis of Population Structure (BAPS) of the data obtained by multilocus sequence typing (MLST) demonstrated that $\mathrm{CC} 17$ may be divided into two subgroups corresponding to major lineages $17 / 18$ and 78 [13].

The vanB gene was identified in E. faecalis at the beginning of the 1990s [14]. In Poland, the first VREfm with vanB2 was isolated in 1999 [15] followed by a growing VanB prevalence in 1999-2005 [16]. A further increase in VanB-VREfm after 2005, noticed by the National Reference Centre for Antimicrobial Resistance and Surveillance (NRCARS), prompted us to investigate these important pathogens to better understand the factors underlying the spread of VanB-E. faecium in Poland.

\section{Materials and methods}

\section{Bacterial isolates and antimicrobial susceptibility testing}

Altogether, 278 non-repetitive isolates with the VanB phenotype received by the NRCARS during 1999-2010 from 36 centers in 22 cities were investigated. Fifty-eight VanB isolates from 1999 to 2005 were partly characterized previously [16]; of these, 56 were available and 222 isolates were received in 2006-2010. Twenty-seven and 48 isolates were obtained from invasive and non-invasive infections, respectively, and 201 from carriage; for two remaining isolates, the source was not reported. Antimicrobial susceptibility was tested using the broth microdilution method [17] and the Etest method for vancomycin, teicoplanin, and daptomycin (bioMerieux, Marcy l'Etoile, France). Results were interpreted following the European Committee on Antimicrobial Susceptibility Testing (EUCAST)-approved breakpoints [18] and the Epidemiological Cut-Offs (ECOFFs) (http://mic.eucast.org/Eucast2/, 6th November 2017, date last accessed).

\section{Detection of $\operatorname{van} B$, IS16 and esp, and molecular typing}

DNA was purified using the Genomic DNA Prep Plus kit (A\&A Biotechnology, Gdynia, Poland) and vanB; IS16 and esp were detected by PCR [19-21]. Multilocus VNTR (variable-number tandem repeat) analysis (MLVA) and MLST were performed as described [22, 23]; sequence types (STs) were assigned using the MLST database http://pubmlst. org/efaecium/ (6th November 2017, date last accessed). On the basis of eBURST analysis [24] of the whole MLST database (as of the 21st of April 2015), STs were included into CCs and lineages $[13,25]$.

\section{Analysis of Tn1549, insertion sites, and coupling sequences}

The presence of $i n t_{\mathrm{Tn} 1549}$ and $\mathrm{ORF} 1_{\mathrm{Tn} 1549}$ was confirmed by PCR, and the van $Y$-van $X$ sequence in Tn1549 was established using overlapping PCR and sequencing. The Tn1549 insertion sites were identified by inverse-PCR (iPCR) [26] with Bsp143I (Fermentas, Lithuania). Primers targeting sequences adjacent to $\operatorname{Tn} 1549$ were designed based on iPCR results. Sequences were analyzed with the Lasergene package (DNASTAR, MD, USA). Primer sequences are available upon request.

\section{Plasmid gene detection, S1 profiles, hybridization, and conjugation}

Plasmid rep $\left(\right.$ rep $1_{\mathrm{pIP} 501}$, rep $2_{\mathrm{pRE} 25}$, rep $8_{\mathrm{pAM} 373}$, rep $9_{\mathrm{pAD} 1}$, rep $\left.17_{\mathrm{pRUM}}, r e p_{\mathrm{pMG} 1}, r e p_{\mathrm{pLG} 1}\right)$ and toxin-antitoxin systems (TAS) axe-txe and $\omega-\varepsilon-\zeta$ were detected by PCR [26-29] with controls from our collection $[28,30]$. For profiling of plasmids, DNA in agarose plugs was treated with S1 nuclease (Takara Bio, Japan), separated by pulsed-gel electrophoresis (PFGE) [31] and blotted onto Hybond-N+ (GE Healthcare, Buckinghamshire, UK). Hybridization was carried out using the Amersham ECL System (GE Healthcare). Transferability of vancomycin resistance was examined as described [32] with the recipient $E$. faecium strain $64 / 3$.

\section{Statistics}

The differences in distributions were evaluated by the chisquared test, with a $p$ value $\leq 0.05$ considered significant.

\section{GenBank accession numbers}

New sequences of the van Y-vanX region: A1-A6 (KC489780KC489785), A9-A20 (KT003969-KT003980), B 1 (KC489787), B2 (KT003981), D (KC489790), and E

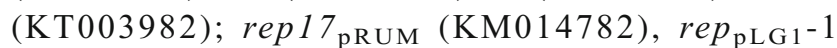
(KM014783), and rep $_{\mathrm{pLG} 1}{ }^{-2}$ (KM014784) were submitted to GenBank.

\section{Results}

\section{Antimicrobial susceptibility phenotypes and clonal relationships of VanB-VREfm in Poland}

All isolates were analyzed by MLVA, yielding 23 different MTs; 13 non-typable isolates repeatedly yielded incomplete MLVA profiles (Table 1). The most prevalent MT159 (186 isolates, $83.0 \%$ ) was observed solely since 2006. Eighty isolates from 2006 to 2010, representing all hospitals providing 


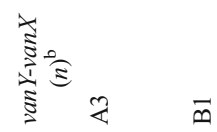

音

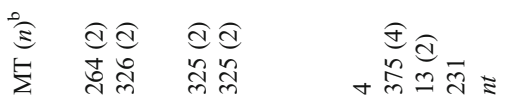

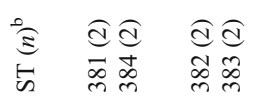

๕ిㅇํำ

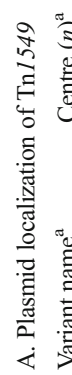

$\underset{\overbrace{}}{0}$

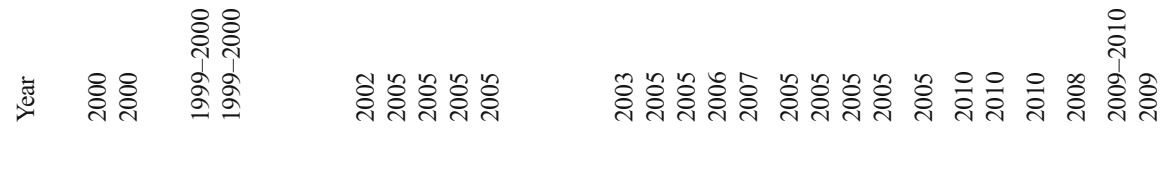

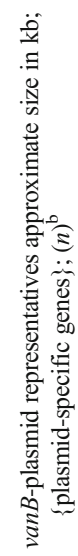

政

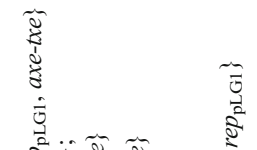

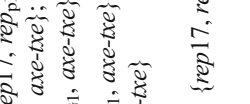

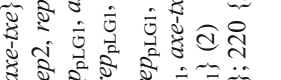

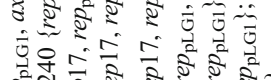

$25=5$

है

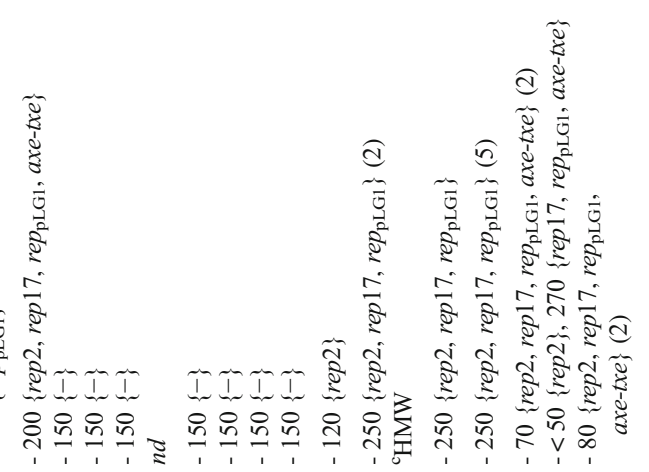

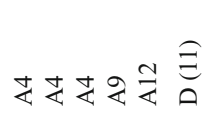

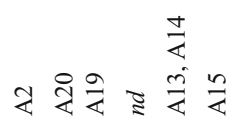

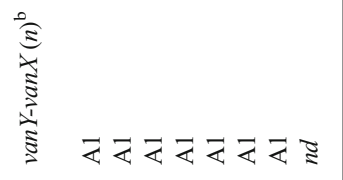

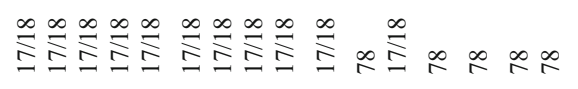

莺

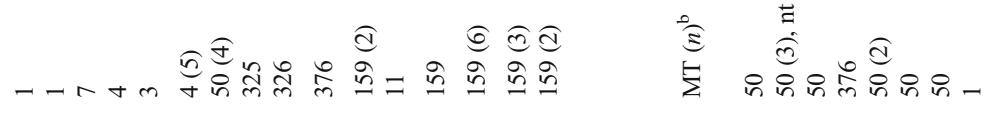

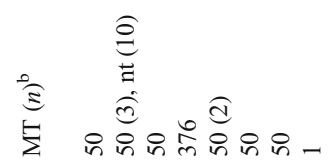

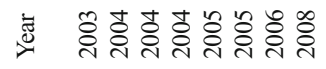

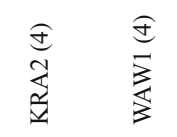
ণิ

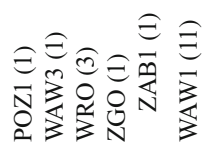

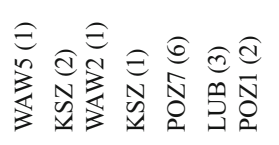

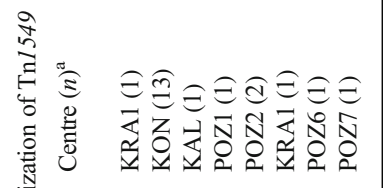
$\begin{array}{lll}\frac{\pi}{a} & \stackrel{0}{a} & \tilde{y}^{\prime} \\ \tilde{y}^{\prime} & \tilde{y}^{\prime} & \tilde{y}^{\prime}\end{array}$
$\begin{array}{ll}m & \vdots \\ \tilde{y}^{\prime} & \tilde{u}^{\prime}\end{array}$

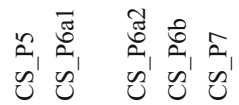

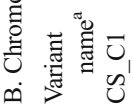

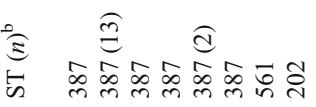




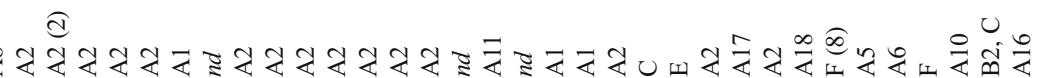

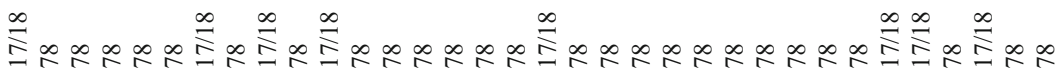

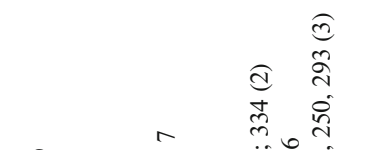

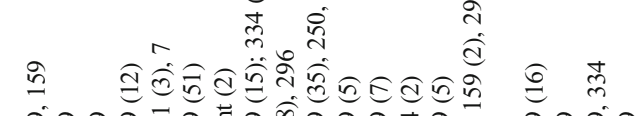

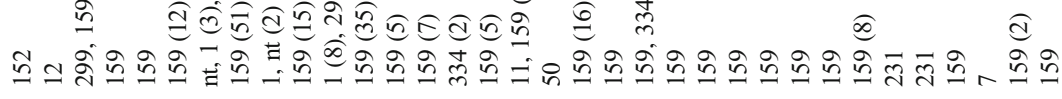

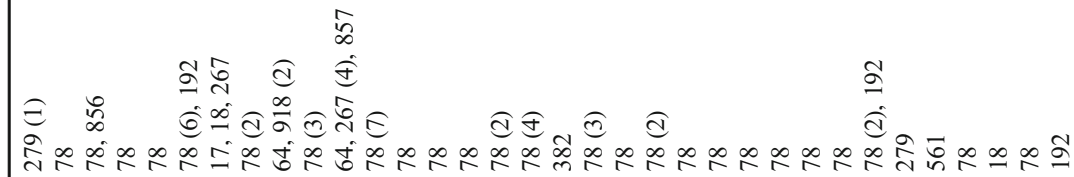

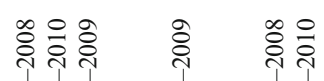

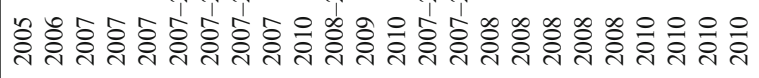

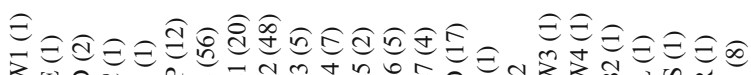

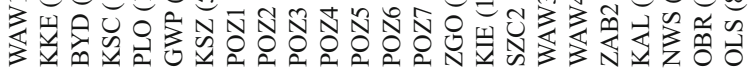

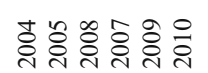

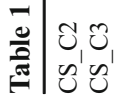

ช

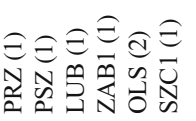

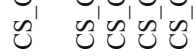

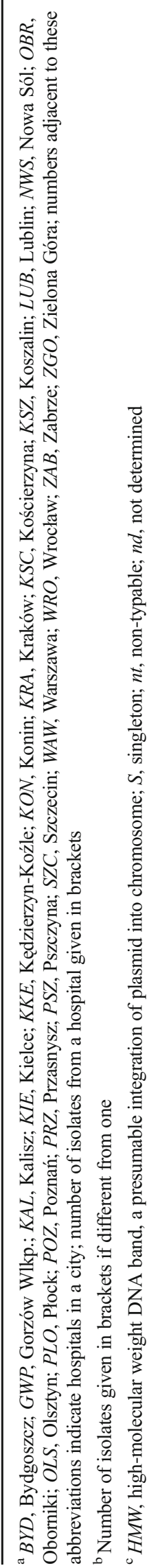


isolates and all MTs, were resistant to ciprofloxacin and ampicillin; 88.8 and $88.8 \%$ isolates showed high-level resistance to gentamicin (HLGR) and streptomycin (HLSR), respectively; $20.0 \%$ of isolates were resistant to tetracycline, which represented a significant decrease $(p=0.0002)$ after $2005(61.5 \%$ [16]). All isolates were susceptible to linezolid, tigecycline, and daptomycin. STs of $26 \mathrm{VanB}$ isolates from the period 1999-2005 were reported previously [16], and additionally 21 isolates from this group were analyzed by MLST, together with 80 representative isolates from the period 2006-2010, mentioned above, yielding altogether 23 STs, characteristic for 127 isolates. Except for ST74, all isolates belonged to lineages $17 / 18$ and 78 , and representatives of 78 lineage were frequently associated with MT159 (Table 1). All isolates carried $v a n B$ and IS16; esp was present in $98 \%$ isolates from 2006 to 2010, similarly to the earlier period [16]. Based on the combined MLVA and MLST results, no representatives of lineage 78 were observed before 2006; the first VanB isolate from lineage 78 occurred in 2006 and since 2007 isolates from this lineage became much more common, representing $89 \%$ of isolates from the period 2006-2010 $(p<0.001)$.

\section{Diversity of the van Y-vanX region in $\operatorname{Tn} 1549$}

All isolates were positive for $\mathrm{ORF} 1_{\mathrm{Tn} 1549}$ and $i n t_{\operatorname{Tn} 1549}$. Sequencing of the van $Y$-vanX region (encompassing genes van $Y$, van $W$, van $H$, van $B$, van $X$; Fig. 1a) revealed 26 variants among 57 isolates, representing all centers and STs within a center. The most numerous group included A1-A20 variants, differing only by single-nucleotide polymorphisms (SNPs) at 21 nucleotide positions and highly similar to the corresponding region in Clostridium spp. and Eggerthella lenta (Fig. 1b). The A-type variants were characteristic for 48 of investigated isolates from 31 centers, and associated with 14 STs and 16 MTs. The B variants differed from the A-type by several SNPs and 6-bp insertion between vanS-vanY. They were $99 \%$ identical to the variant reported for the V583 [33]. An insertion of the ISEfall between vanS-vanY in B-type yielded C variants (Fig. 1a). The D-, E-, and F-types represented probable derivatives of an A-type, with a deletion encompassing the nt 12799 of vanW, an 11-bp deletion upstream van $Y$, and insertion of IS $L 3$ between vanS and van $Y$, respectively. All vanB genes represented the vanB2 variant [34].

\section{Analysis of Tn1549 insertion sites and coupling sequences}

To determine Tn1549 insertion sites, selected isolates were analyzed by iPCR and thus obtained sequences were used to search GenBank and to design primers specific for a genetic neighborhood of $\operatorname{Tn} 1549$. These primers were used to screen the whole collection, revealing 15 insertion sites and 14 coupling sequences in total (Table 2). For two isolates, the coupling sequence could not be established due to the fact that sequences resulting from iPCR had no homologs in GenBank. Typically coupling sequences were identical in a given insertion site, with an exception of CS P1a/CS P1b in aacA-aphD and CS P6a/CS P6b in citH. The most prevalent coupling sequence, CS_C3 (198 isolates from 24 centers in 16 cities) was associated with 16 MTs and 14 STs. The first CS_C3 isolate was observed in 2006 (Table 1B).

\section{Analysis of Tn1549 localization, plasmidome composition, and van $B$ transferability}

Seventy-eight isolates were analyzed by S1/PFGEhybridization with the $\operatorname{van} B$ probe (Table 1 and Supplementary Fig. 1). These isolates represented all observed variants of coupling sequence and hospital centers; additional isolates from the same center were included in the case of isolates showing plasmid localization of $\operatorname{vanB}$. In the case of 39 isolates with coupling sequence $\mathrm{C} 1-\mathrm{C} 8$, vanB hybridized with a band of high-molecular weight, consistent with transposon insertion within chromosomal sequences and 39 isolates showed hybridization with plasmids from 30 to $\sim 310 \mathrm{~kb}$ in size; in five isolates, $\operatorname{van} B$ was located on two plasmids. These hybridization studies and iPCR/PCRbased analyses of coupling sequences were consistent with the chromosomal localization of $\operatorname{Tn} 1549$ for 227 isolates (81.6\%) and plasmid localization for 50 isolates (18.0\%); in a single case, a presumable integration of plasmid into chromosome was observed (variant CS_6al from WAW2). Isolates with the plasmid localization of $\operatorname{van} B$ were much more prevalent among early VREfm, i.e., from 1999 to 2005 (61\% of these isolates) compared to the isolates collected from 2006 to 2010 ( $0.7 \%$ of these isolates, $p<0.001)$. Among isolates with the plasmid localization of vanB, rep $17_{\mathrm{pRUM}}$ was found among 49 isolates, followed by $r e p_{\mathrm{pLG} 1}$, rep $2_{\mathrm{pRE} 25}$, $r e p 1_{\mathrm{pIP} 501}, r e p_{\mathrm{pMG} 1}$, and rep $9_{\mathrm{pAD} 1}(42,35,32,27$, and 5 isolates, respectively). Thirty-one and 12 of these isolates carried axe-txe and $\omega-\varepsilon-\zeta)$, respectively. S1-PFGE/hybridization analyses revealed that 29,23 , and 22 plasmids hybridized with the rep $p_{\mathrm{pLG} 1}$, rep $17_{\mathrm{pRUM}}$, and rep $2_{\mathrm{pRE} 25}$ probes, respectively (Table 1A). In several cases, a single plasmid was associated with two or three rep genes. Sixteen vanB-plasmids hybridized with the axe-txe probe; among them, 13 co-hybridized with rep $_{\mathrm{pLG} 1}$ and 12 with rep $17_{\mathrm{pRUM}}$, respectively. Nine plasmids did not hybridize with any of the four probes used. Sequencing revealed a low variability of rep genes within this group that included two, one, three, one, and three variants of $r e p 1_{\mathrm{pIP} 501}, r e p 2_{\mathrm{pRE} 25}, r e p 17_{\mathrm{pRUM}}, r e p_{\mathrm{pMG}}$, and $r e p_{\mathrm{pLG} 1}$, respectively. Among 50 isolates with the plasmid localization of vanB, 43 isolates $(86.0 \%)$ were able to transfer vancomycin resistance while conjugation experiments involving 32 representative isolates with various chromosomal localizations of van $B$ were negative in 29 cases. 
Fig. 1 Diversity of van Y-vanX region among VanB-VREfm in Poland, 1998-2010. a Structure of the region, distribution of single-nucleotide polymorphisms (marked by asterisks) among Atype variants, and localization of deletions and ISs. b Similarity tree of nucleotide sequences of Aand B-type variants and sequences from the V583 strain of E. faecalis and isolates of E. lenta and Clostridium spp

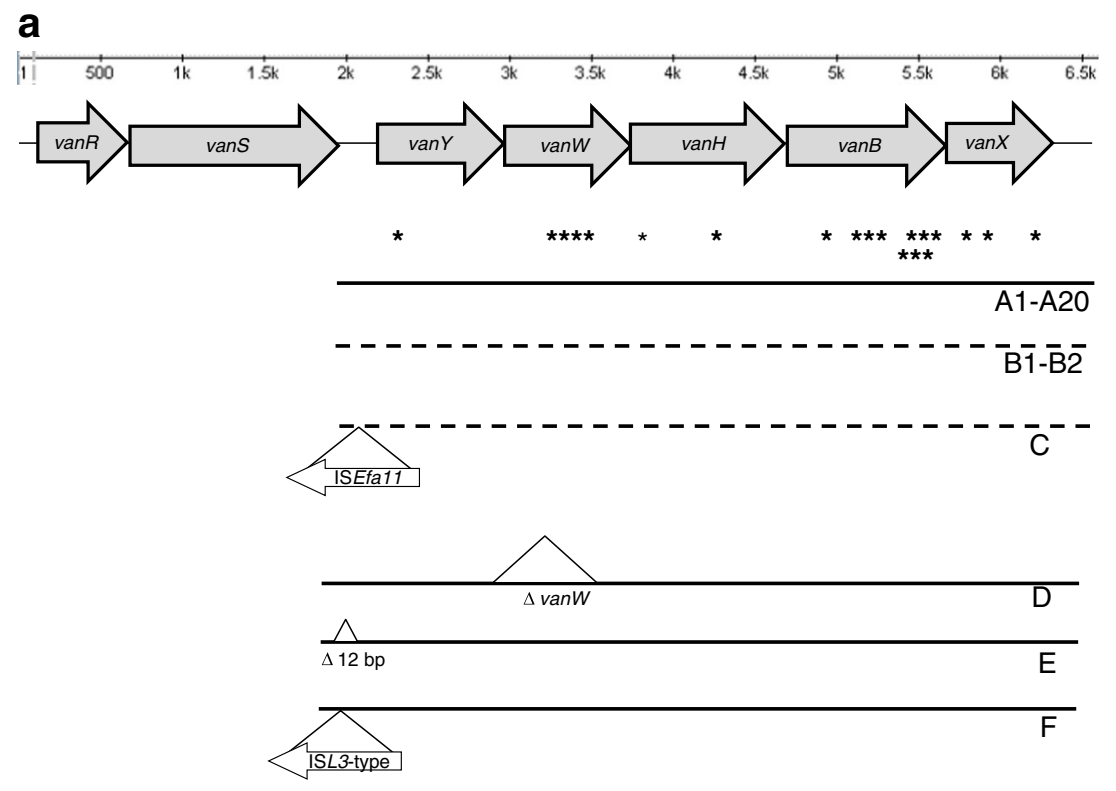

b

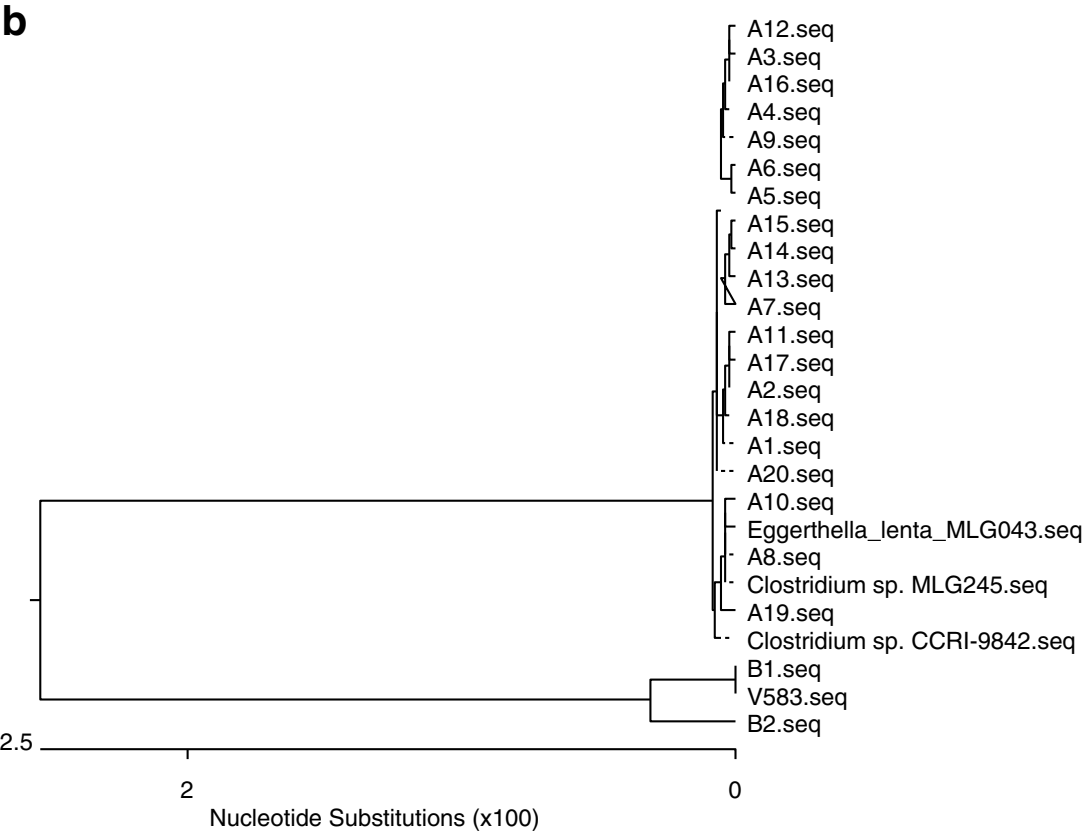

\section{Discussion}

The first VanB-VREfm was detected in Poland in 1999 [15], and our study investigated the VanB epidemiology during the following 12 years. Considering a relatively moderate incidence of VREfm in Poland during this period (e.g., in 2010 amounting to $7.8 \%$ of invasive infections [http://ecdc.europa.eu/en/publications/Publications/1111_ SUR AMR data.pdf.pdf; 6th November 2017, date last accessed]), it may be assumed that our collection reasonably well reflected the epidemiological situation in Polish hospitals. Although initially VanA represented the major VREfm phenotype in Poland [16, 35], after 2006, the
NRCARS recorded an increasing number of VanB-VREfm, affecting several hospitals. The current global epidemiology of VREfm shows considerable differences, with VanA predominant in Europe and the USA [36], and VanB constituting over $80 \%$ of invasive VREfm in Australia [37]. A recent rise of VanB-E. faecium has been reported in Germany [8]. Nearly all isolates in our study belonged to the hospital E. faecium, since 2006 with the predominant role $(89 \%)$ of lineage 78 . VanB-VREfm belonging to this lineage were responsible for recent outbreaks in Germany, Sweden, and Australia [38], and representatives of lineage 78 played a role in van $A$ dissemination in Polish hospitals [34]. 


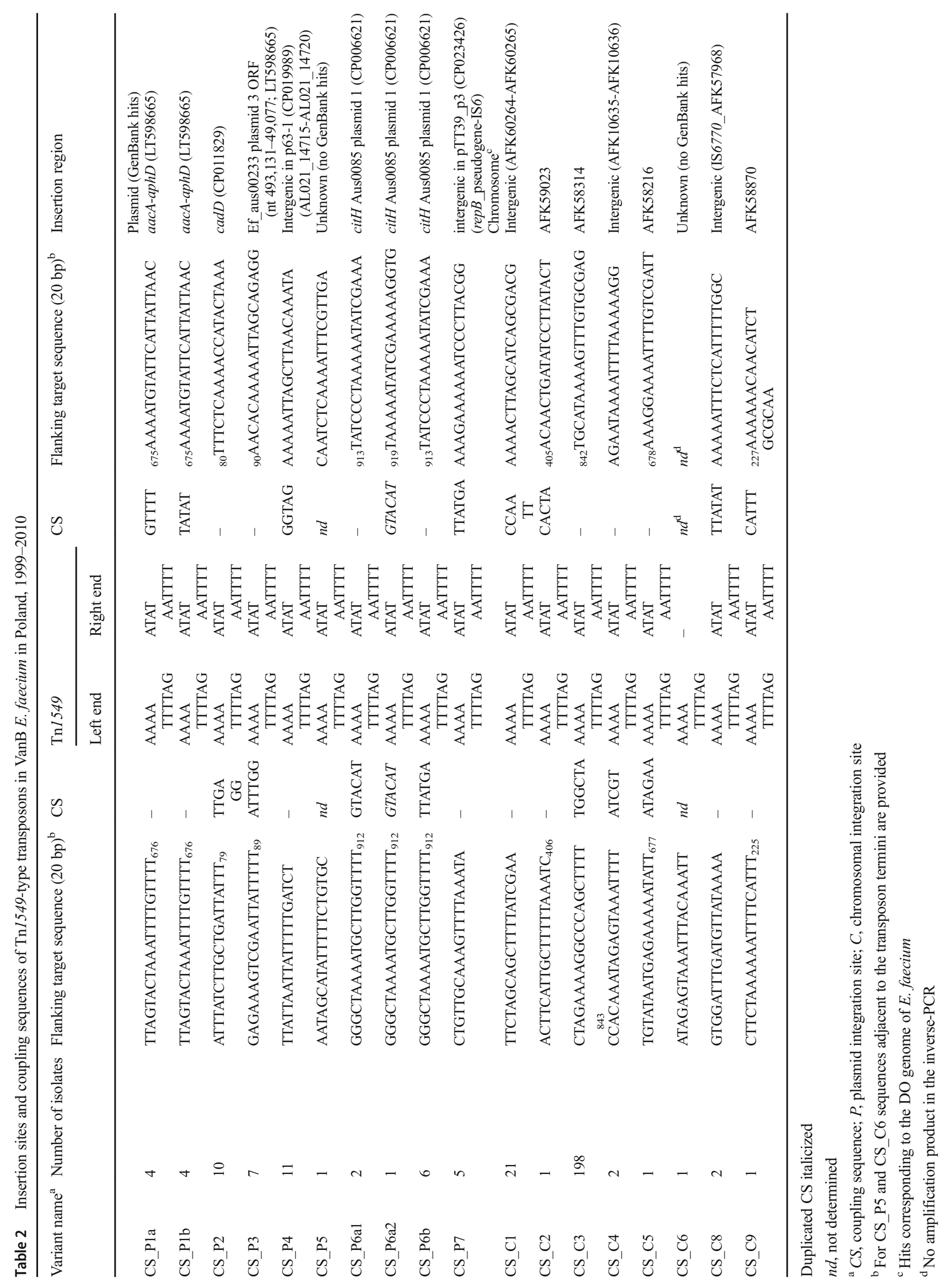


While the structure of $\operatorname{Tn} 1546$, harboring vanA shows a high variability $[34,39]$, the van $Y$-van $X$ region in the vanB gene cluster appeared to be less divergent. In the studied collection, the A-type showed the highest prevalence, with variants very similar or identical to these found in E. faecium in Australia, France, and Taiwan [40-42], and in the pMG2200 plasmid of E. faecalis [5]. Importantly, the A variants are also present in gut anaerobes such as Clostridium spp. and E. lenta [26, 40], a presumable reservoir of Tn1549-type transposons. Genomic analyses of VanB-E. faecium and concomitantly isolated vanB-positive gut anaerobes indicated the epidemiological significance of de novo acquisition of $\operatorname{Tn} 1549$ by hospital-adapted E. faecium [7, 41]. The B-type characteristic for the first vancomycin-resistant $E$. faecalis V583 strain [33], to our knowledge, has not been reported in E. faecium so far. The presence of ISs targeting the vanS-van $Y$ intergenic region (resulting in C- and F-types), was observed also elsewhere [43]. Such variability of $v a n B$ clusters may be useful in analyses of suspected VRE outbreaks. For example, plasmid-located Dtype was found in isolates representing various MTs and STs from the WAW1 hospital (Table 1A). Thus, a spread of a stable $\sim 150 \mathrm{~kb}$ conjugative plasmid of undetermined replicon type, harboring this specific variant of the $\operatorname{van} B$ cluster was most likely responsible for the outbreak. Similarly, although isolates from SZC2 differed both in the clonal composition and vanB-associated plasmidome, C-type was detected in all these isolates (Table 1A), indicating extensive plasmid recombination during an outbreak. Until now, more detailed knowledge of plasmids carrying vanB in E. faecium remains limited [38]. In our study, vanB-plasmids represented mostly the rep $p_{\mathrm{pLG} 1}$, rep $17_{\mathrm{pRUM}}$, and rep $2_{\mathrm{pRE} 25}$ replicons, similarly to the situation observed for vanA-plasmids in Poland [34]. The original pLG1 contained the complete vanA gene cluster [44] and plasmids with this rep were responsible for an increase of HLGR among E. faecium in Norway [45] but, to our knowledge, vanB-plasmid of the rep $p_{\mathrm{pLG} 1}$ type has not yet been reported. The second observed rep type, rep 17 pRUM was involved in a multicenter VanB outbreak in Sweden [46] and in the HLGR spread in Norway [45]. The rep $_{\mathrm{pLG} 1}$ and rep $17_{\mathrm{pRUM}}$ genes frequently occurred together and in combination with the axe-txe, characteristic for plasmids with these replicons [34, 45, 46]. Plasmids harboring vanB were typically transferable by conjugation and during outbreaks (e.g., in KRA2, WAW1 and SZC2) were associated with diverse clonal backgrounds. Such plasmid dissemination was additionally accompanied by presumable recombination/co-integration events, resulting in the observed variability of vanB-plasmids. A similar dynamics was observed

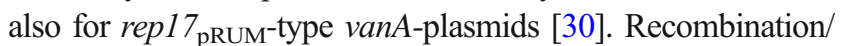
co-integration likely contributed to the association of $\operatorname{van} B$ with more than a single rep, observed in the current study and characteristic for E. faecium plasmids in general [30, 34, 38, 45]. Two plasmid-located genes, aacA-aphD and $c i t H$, showed the integration of Tn1549 with different coupling sequences and might represent transposon integration hotspots. Such hotspots were indeed observed for E. faecium [7].

Isolates with plasmid-borne vanB prevailed until 2006, and later this determinant showed usually a chromosomal localization. This change occurred in parallel with the emergence and spread of lineage 78 . The predominantly chromosomal localization of the vanB cluster in lineage 78 was observed recently also in Germany and Australia [7, 8, 40]. Two variants of coupling sequences, CS_C1 and CS_C3, were associated with two most numerous groups of isolates (Table 1). Twenty-one isolates with the CS_C1 variant, present in 17/ 18 lineage and A1 type of the van $Y$-vanX region, showed multicenter distribution over 2003-2008. These isolates showed some divergence of their STs/MTs, which may be explained by a transfer of transposon-containing region to a new clonal background [8] and/or exchange of other genomic regions, leading to formation of new STs/MTs [9]. An even more complex epidemiological situation was associated with isolates harboring the CS_C3 variant. This particular group appears to be the main contributor to the increasing proportion of VanB among VREfm and general increase of prevalence of VRE in Poland and was responsible for extensive outbreaks, e.g., in KSZ and POZ2 hospitals. With the exception of a single isolate with CS_C5, which shared a coupling sequence and insertion site with several ST192 isolates from Germany [8], none of the remaining coupling sequences showed identity to coupling sequences described elsewhere [7-9]. This finding is consistent with proposed independent de novo acquisition of $\operatorname{Tn} 1549$ [7].

This study provides an analysis of VanB-E. faecium, performed on a country level and over an extensive period of time. We demonstrate a significant change both in the clonal background as well as localization of Tn1549-type transposons, carrying vanB genes. Our study supports the role of lineage 78 of the hospital-adapted E. faecium, presumably acquiring de novo the van $B$ determinant, followed by spread and differentiation of certain strains as a major factor beyond the current increasing prevalence of VanB-VREfm in Polish hospitals.

Acknowledgements We thank all microbiologists who provided isolates to the National Reference Centre for Antimicrobial Resistance and Surveillance in Warsaw and Dr. Kenneth Van Horn for a critical reading of the manuscript. This publication made use of the Enterococcus faecium MLST website (http://pubmlst.org/efaecium/) sited at the University of Oxford and funded by the Wellcome Trust.

[Parts of this study were presented at the Applied Bioinformatics \& Public Health Microbiology Conference, 15-17 May 2013, Cambridge, UK; the Microbiology after genomic revolution: Genomes 2014 EMBO Conference, 24-27 June 2014, Paris, France and the International Conference on the Evolution and Transfer of Antibiotic Resistance, 2426 June 2015, Amsterdam, The Netherlands.]

Funding This work was supported by grant N N401588540 from the National Science Centre (NCN), Poland, by the MIKROBANK funding from the Ministry of Science and Higher Education, Poland, and by 
statutory funds from the Ministry of Science and Higher Education, Poland.

\section{Compliance with ethical standards}

Isolates were obtained as a part of routine activity of the NRCARS and were analyzed anonymously in a retrospective manner. Ethical approval and informed consent were thus not required.

Conflict of interest The authors declare that they have no conflict of interest.

Open Access This article is distributed under the terms of the Creative Commons Attribution 4.0 International License (http:// creativecommons.org/licenses/by/4.0/), which permits unrestricted use, distribution, and reproduction in any medium, provided you give appropriate credit to the original author(s) and the source, provide a link to the Creative Commons license, and indicate if changes were made.

\section{References}

1. Sydnor ER, Perl TM (2011) Hospital epidemiology and infection control in acute-care settings. Clin Microbiol Rev 24:141-173. https://doi.org/10.1128/CMR.00027-10

2. Arias CA, Murray BE (2008) Emergence and management of drugresistant enterococcal infections. Exp Rev Anti Infect Ther 6:637655. https://doi.org/10.1586/14787210.6.5.637

3. Courvalin P (2006) Vancomycin resistance in Gram-positive cocci. Clin Infect Dis 42(Suppl 1):S25-S34. https://doi.org/10.1086/ 491711

4. Garnier F, Taourit S, Glaser P, Courvalin P, Galimand M (2000) Characterization of transposon $\operatorname{Tn} 1549$, conferring VanB-type resistance in Enterococcus spp. Microbiology 146:1481-1489. https://doi.org/10.1099/00221287-146-6-1481

5. Zheng B, Tomita H, Inoue T, Ike Y (2009) Isolation of VanB-type Enterococcus faecalis strains from nosocomial infections: first report of the isolation and identification of the pheromone-responsive plasmids pMG2200, encoding VanB-type vancomycin resistance and a Bac41-type bacteriocin, and pMG2201, encoding erythromycin resistance and cytolysin (Hly/Bac). Antimicrob Agents Chemother 53:735-747. https://doi.org/10.1128/AAC.00754-08

6. Bjørkeng E, Rasmussen G, Sundsfjord A, Sjöberg L, Hegstad K, Söderquist B (2011) Clustering of polyclonal VanB-type vancomycin-resistant Enterococcus faecium in a low-endemic area was associated with CC17-genogroup strains harbouring transferable vanB2-Tn5382 and pRUM-like repA containing plasmids with axe-txe plasmid addiction systems. APMIS 119:247-258. https:// doi.org/10.1111/j.1600-0463.2011.02724

7. Howden BP, Holt KE, Lam MM, Seemann T, Ballard S, Coombs GW, Tong SY, Grayson ML, Johnson PD, Stinear TP (2013) Genomic insights to control the emergence of vancomycinresistant enterococci. MBio 4:e00412-e00413. https://doi.org/10. 1128/mBio.00412-13

8. Bender JK, Kalmbach A, Fleige C, Klare I, Fuchs S, Werner G (2016) Population structure and acquisition of the $\operatorname{van} B$ resistance determinant in German clinical isolates of Enterococcus faecium ST192. Sci Rep 6:21847. https://doi.org/10.1038/srep21847

9. van Hal SJ, Ip CL, Ansari MA, Wilson DJ, Espedido BA, Jensen SO, Bowden R (2016) Evolutionary dynamics of Enterococcus faecium reveals complex genomic relationships between isolates with independent emergence of vancomycin resistance. Microb Genom 2. doi: https://doi.org/10.1099/mgen.0.000048

10. Edelsberg J, Weycker D, Barron R, Li X, Wu H, Oster G, Badre S, Langeberg WJ, Weber DJ (2014) Prevalence of antibiotic resistance in US hospitals. Diagn Microbiol Infect Dis 78:255-262. https:// doi.org/10.1016/j.diagmicrobio.2013.11.011

11. de Kraker ME, Jarlier V, Monen JC, Heuer OE, van de Sande N, Grundmann H (2013) The changing epidemiology of bacteraemias in Europe: trends from the European Antimicrobial Resistance Surveillance System. Clin Microbiol Infect 19:860-868. https:// doi.org/10.1111/1469-0691.12028

12. Willems RJ, Top J, van Santen M, Robinson DA, Coque TM, Baquero F, Grundmann H, Bonten MJ (2005) Global spread of vancomycin-resistant Enterococcus faecium from distinct nosocomial genetic complex. Emerg Infect Dis 11:821-828. https://doi. org/10.3201/eid1106.041204

13. Willems RJ, Top J, van Schaik W, Leavis H, Bonten M, Sirén J, Hanage WP, Corander J (2012) Restricted gene flow among hospital subpopulations of Enterococcus faecium. MBio 3:e0151e00112. https://doi.org/10.1128/mBio.00151-12

14. Evers S, Sahm DF, Courvalin P (1993) The vanB gene of vancomycin-resistant Enterococcus faecalis V583 is structurally related to genes encoding D-Ala:D-Ala ligases and glycopeptideresistance proteins VanA and VanC. Gene 124:143-144. https:// doi.org/10.1016/0378-1119(93)90779-3

15. Kawalec M, Gniadkowski M, Zielińska U, Kłos W, Hryniewicz W (2001) Vancomycin-resistant Enterococcus faecium strain carrying the vanB2 gene variant in a Polish hospital. J Clin Microbiol 39: 811-815. https://doi.org/10.1128/JCM.39.2.811-815.2001

16. Sadowy E, Sieńko A, Gawryszewska I, Bojarska A, Malinowska K, Hryniewicz W (2013) High abundance and diversity of antimicrobial resistance determinants among early vancomycin-resistant Enterococcus faecium in Poland. Eur J Clin Microbiol Infect Dis 32:1193-1203. https://doi.org/10.1007/s10096-013-1868-y

17. Clinical and Laboratory Standards Institute (2010) Performance standards for antimicrobial susceptibility testing; 20th informational supplement 2010; Wayne, PA M100-S20

18. The European Committee on Antimicrobial Susceptibility Testing. Breakpoint tables for interpretation of MICs and zone diameters. Version 7.1, 2017. http://www.eucast.org/fileadmin/src/media/ PDFs/EUCAST_files/Breakpoint_tables/v_7.1_Breakpoint_ Tables.pdf. $6^{\text {th }}$ November 2017, date last accessed

19. Klare I, Konstabel C, Mueller-Bertling S, Werner G, Strommenger B, Kettlitz C, Borgmann S, Schulte B, Jonas D, Serr A, Fahr AM, Eigner U, Witte W (2005) Spread of ampicillin/vancomycinresistant Enterococcus faecium of the epidemic-virulent clonal complex-17 carrying the genes esp and hyl in German hospitals. Eur J Clin Microbiol Infect Dis 24:815-825. https://doi.org/10. 1007/s10096-005-0056-0

20. Werner G, Fleige C, Geringer U, van Schaik W, Klare I, Witte W (2011) IS element IS16 as a molecular screening tool to identify hospital-associated strains of Enterococcus faecium. BMC Infect Dis 31:80. https://doi.org/10.1186/1471-2334-11-80

21. Vankerckhoven V, Van Autgaerden T, Vael C, Lammens C, Chapelle S, Rossi R, Jabes D, Goossens H (2004) Development of a multiplex PCR for the detection of asa 1, gelE, cylA, esp and hyl genes in enterococci and survey for virulence determinants among European hospital isolates of Enterococcus faecium. J Clin Microbiol 42:4473-4479. https://doi.org/10.1128/JCM.42.10. 4473-4479.2004

22. Top J, Schouls LM, Bonten MJ, Willems RJ (2004) Multiple-locus variable-number tandem repeat analysis, a novel typing scheme to study the genetic relatedness and epidemiology of Enterococcus faecium isolates. J Clin Microbiol 42:4305-4511. https://doi.org/ 10.1128/JCM.42.10.4503-4511.2004 
23. Homan WL, Tribe D, Poznanski S, Li M, Hogg G, Spalburg E, Van Embden JD, Willems RJ (2002) Multilocus sequence typing scheme for Enterococcus faecium. J Clin Microbiol 40:19631971. https://doi.org/10.1128/JCM.40.6.1963-1971.2002

24. Feil EJ, Li BC, Aanensen DM, Hanage WP, Spratt BG (2004) eBURST: inferring patterns of evolutionary descent among clusters of related bacterial genotypes from multilocus sequence typing data. J Bacteriol 186:1518-1530. https://doi.org/10.1128/JB.186.5. 1518-1530.2004

25. Freitas AR, Novais C, Ruiz-Garbajosa P, Coque TM, Peixe L (2009) Dispersion of multidrug-resistant Enterococcus faecium isolates belonging to major clonal complexes in different Portuguese settings. Appl Environ Microbiol 75:4904 4908. https://doi.org/10. 1128/AEM.02945-08

26. Launay A, Ballard SA, Johnson PD, Grayson ML, Lambert T (2006) Transfer of vancomycin resistance transposon Tn1549 from Clostridium symbiosum to Enterococcus spp. in the gut of gnotobiotic mice. Antimicrob Agents Chemother 50:1054-1062. https:// doi.org/10.1128/AAC.50.3.1054-1062.2006

27. Jensen LB, Garcia-Migura L, Valenzuela AJ, Løhr M, Hasman H, Aarestrup FM (2010) A classification system for plasmids from enterococci and other Gram-positive bacteria. J Microbiol Methods 80:25-43. https://doi.org/10.1016/j.mimet.2009.10.012

28. Wardal E, Gawryszewska I, Hryniewicz W, Sadowy E (2013) Abundance and diversity of plasmid-associated genes among clinical isolates of Enterococcus faecalis. Plasmid 70:329-342. https:// doi.org/10.1016/j.plasmid.2013.07.003

29. Moritz EM, Hergenrother PJ (2007) Toxin-antitoxin systems are ubiquitous and plasmid-encoded in vancomycin-resistant enterococci. Proc Natl Acad Sci U S A 104:311-316. https://doi.org/10. 1073/pnas.0601168104

30. Wardal E, Markowska K, Zabicka D, Wróblewska M, Giemza M, Mik E, Połowniak-Pracka H, Woźniak A, Hryniewicz W, Sadowy E (2014) Molecular analysis of vanA outbreak of Enterococcus faecium in two Warsaw hospitals: the importance of mobile genetic elements. Biomed Res Int 2014:575367. https://doi.org/10.1155/ 2014/575367

31. Barton BM, Harding GP, Zuccarelli AJ (1995) A general method for detecting and sizing large plasmids. Anal Biochem 226:235240. https://doi.org/10.1006/abio.1995.1220

32. Manson JM, Hancock LE, Gilmore MS (2010) Mechanism of chromosomal transfer of Enterococcus faecalis pathogenicity island, capsule, antimicrobial resistance, and other traits. Proc Natl Acad Sci U S A 107:12269-12274. https://doi.org/10.1073/pnas. 1000139107

33. Paulsen IT, Banerjei L, Myers GS, Nelson KE, Seshadri R, Read TD, Fouts DE, Eisen JA, Gill SR, Heidelberg JF, Tettelin H, Dodson RJ, Umayam L, Brinkac L, Beanan M, Daugherty S, DeBoy RT, Durkin S, Kolonay J, Madupu R, Nelson W, Vamathevan J, Tran B, Upton J, Hansen T, Shetty J, Khouri H, Utterback T, Radune D, Ketchum KA, Dougherty BA, Fraser CM (2003) Role of mobile DNA in the evolution of vancomycinresistant Enterococcus faecalis. Science 299:2071-2074. https:// doi.org/10.1126/science.1080613

34. Patel R, Uhl JR, Kohner P, Hopkins MK, Steckelberg JM, Kline B, Cockerill FR 3rd (1998) DNA sequence variation within vanA, van $B$, van $C$-1, and vanC-2/3 genes of clinical Enterococcus isolates. Antimicrob Agents Chemother 42:202-205

35. Wardal E, Kuch A, Gawryszewska I, Żabicka D, Hryniewicz W, Sadowy E (2017) Diversity of plasmids and Tn1546-type transposons among VanA Enterococcus faecium in Poland. Eur J Clin
Microbiol Infect Dis 36:313-328. https://doi.org/10.1007/s10096016-2804-8

36. Deshpande LM, Fritsche TR, Moet GJ, Biedenbach DJ, Jones RN (2007) Antimicrobial resistance and molecular epidemiology of vancomycin-resistant enterococci from North America and Europe: a report from the SENTRY antimicrobial surveillance program. Diagn Microbiol Infect Dis 58:163-170. https://doi.org/10. 1016/j.diagmicrobio.2006.12.022

37. Coombs GW, Daley DA, Thin Lee Y, Pang S, Pearson JC, Robinson JO, Johnson PD, Kotsanas D, Bell JM, Turnidge JD, Australian Group on Antimicrobial Resistance (2016) Australian group on antimicrobial resistance Australian enterococcal sepsis outcome programme annual report, 2014. Commun Dis Intell Q Rep 40:E236-E243

38. Freitas AR, Tedim AP, Francia MV, Jensen LB, Novais C, Peixe L, Sánchez-Valenzuela A, Sundsfjord A, Hegstad K, Werner G, Sadowy E, Hammerum AM, Garcia-Migura L, Willems RJ, Baquero F, Coque TM (2016) Multilevel population genetic analysis of vanA and vanB Enterococcus faecium causing nosocomial outbreaks in 27 countries (1986-2012). J Antimicrob Chemother 71:3351-3366. https://doi.org/10.1093/jac/dkw312

39. Willems RJ, Top J, van den Braak N, van Belkum A, Mevius DJ, Hendriks G, van Santen-Verheuvel M, van Embden JD (1999) Molecular diversity and evolutionary relationships of Tn1546-like elements in enterococci from humans and animals. Antimicrob Agents Chemother 43:483-491

40. Ballard SA, Pertile KK, Lim M, Johnson PD, Grayson ML (2005) Molecular characterization of vanB elements in naturally occurring gut anaerobes antimicrob. Agents Chemother 49:1688-1694. https://doi.org/10.1128/AAC.49.5.1688-1694.2005

41. Lam MM, Seemann T, Bulach DM, Gladman SL, Chen H, Haring V, Moore RJ, Ballard S, Grayson ML, Johnson PD, Howden BP, Stinear TP (2012) Comparative analysis of the first complete Enterococcus faecium genome. J Bacteriol 194:2334-2341. https://doi.org/10.1128/JB.00259-12

42. Lu JJ, Perng CL, Ho MF, Chiueh TS, Lee WH (2001) High prevalence of VanB2 vancomycin-resistant Enterococcus faecium in Taiwan. J Clin Microbiol 39:2140-2145. https://doi.org/10.1128/ JCM.39.6.2140-2145.2001

43. Dahl KH, Lundblad EW, Rokenes TP, Olsvik O, Sundsfjord A (2000) Genetic linkage of the vanB2 gene cluster to Tn5382 in vancomycin-resistant enterococci and characterization of two novel insertion sequences. Microbiology 146:1469-1479. https://doi.org/ 10.1099/00221287-146-6-1469

44. Laverde Gomez JA, van Schaik W, Freitas AR, Coque TM, Weaver KE, Francia MV, Witte W, Werner G (2011) A multiresistance megaplasmid pLG1 bearing a $h y l_{\text {Efm }}$ genomic island in hospital Enterococcus faecium isolates. Int J Med Microbiol 301:165-175. https://doi.org/10.1016/j.ijmm.2010.08.015

45. Rosvoll TC, Lindstad BL, Lunde TM, Hegstad K, Aasnaes B, Hammerum AM, Lester CH, Simonsen GS, Sundsfjord A, Pedersen T (2012) Increased high-level gentamicin resistance in invasive Enterococcus faecium is associated with aac(6')Ieaph (2")Ia-encoding transferable megaplasmids hosted by major hospital-adapted lineages. FEMS Immunol Med Microbiol 66: 166-176. https://doi.org/10.1111/j.1574-695X.2012.00997.x

46. Sivertsen A, Billström H, Melefors O, Liljequist BO, Wisell KT, Ullberg M, Ozenci V, Sundsfjord A, Hegstad K (2014) A multicentre hospital outbreak in Sweden caused by introduction of a vanB2 transposon into a stably maintained pRUM-plasmid in an Enterococcus faecium ST192 clone. PLoS One 9:e103274. https://doi.org/10.1371/journal.pone.0103274 